\title{
EFEITO DO TREINAMENTO MUSCULAR INSPIRATÓRIO NA FUNÇÃO PULMONAR E ESTADO FUNCIONAL DE UM PACIENTE COM ANEMIA FALCIFORME: RELATO DE CASO
}

\author{
Érica Mirales Rodrigues*, Leilane Rebouças Cardoso*, Mila Vaz C. S. Oliva**, Bruno Prata Martinez***, \\ Thiago Queiroz Pires**** \\ Autor correspondente: Bruno Prata Martinez. Email: brunopmartinez@hotmail.com \\ * Graduanda do curso de Fisioterapia da União Metropolitana de Educação e Cultura \\ ** Professora do curso de Fisioterapia da União Metropolitana de Educação e Cultura \\ *** Professor Auxiliar da Universidade do Estado da Bahia. Fisioterapeuta Líder da UTI do Hospital Aliança. Doutorando em \\ Medicina e Saúde Humana pela Escola Bahiana de Medicina e Saúde Pública \\ **** Fisioterapeuta da Reative Fisioterapia Especializada. Especialista em Fisioterapia Cardiorrespiratória pela UGF
}

\section{Resumo}

Objetivo: Este artigo relata o caso de um indivíduo adulto com diagnóstico de anemia falciforme (AF) desde a adolescência, com histórico de várias internações hospitalares ao longo dos anos e com relato de dispnéia durante algumas atividades de vida diária. Descrição do caso: $\mathrm{Na}$ avaliação inicial foram obtidos os valores de pressão inspiratória máxima (PImáx) e da capacidade vital (CV) através do aparelho POWERbreathe $®$ digital modelo K5 (POWERbreathe International Limited, Londres, Inglaterra), e do índice basal de dispnéia (BDI) durante as atividades de vida diária. Após a identificação da fraqueza muscular inspiratória (PImáx $\leq 60 \%$ do predito), foi sugerido a realização do treinamento muscular inspiratório (TMI) com um dispositivo resistor inspiratório de carga linear. O ajuste inicial foi com uma carga de $65 \%$ da PImáx, durante 6 semanas, de forma diária, com 30 incursões realizadas duas vezes ao dia. No $21^{\circ}$ e $42^{\circ}$ dia foram realizadas novas avaliações da PImáx e CV, sendo reavaliado o BDI no $42^{\circ}$ dia. No $21^{\circ}$ dia do programa foi verificado uma elevação de $13,8 \%$ na PImáx e $82,3 \%$ na CV. Já no $42^{\circ}$ dia, foi observado mais um ganho na PImáx sem alteração dos valores da CV. $\mathrm{Na}$ análise do BDI foi verificado um incremento de 7 para 12 pontos $(71,4 \%)$, o que caracteriza um paciente sem dispnéia ao realizar as atividades propostas pelo instrumento. Conclusão: Identificou-se uma melhora da força muscular inspiratória e da dispnéia nas atividades diárias após o programa de TMI no presente relato de caso.

Palavras-chave: Anemia Falciforme; Função pulmonar; Treinamento muscular inspiratório. 


\title{
EFFECT OF INSPIRATORY MUSCLE TRAINING ON LUNG FUNCTION AND FUNCTIONAL STATUS OF A PATIENT WITH SICKLE CELL DISEASE: CASE REPORT
}

\begin{abstract}
Objective: This article reports the case of an adult diagnosed with sickle cell anemia (SCA) since adolescence, with a history of multiple hospitalizations over the years and dyspnea report during some activities of daily living. Case description: In the initial evaluation was obtained the maximal inspiratory pressure (MIP) and vital capacity (VC) through POWERbreathe $®$ digital device $\mathrm{K}_{5}$ model (POWERbreathe International Limited, London, England), and baseline dyspnea index (BDI) during activities of daily living. After the diagnosis of inspiratory muscle weakness (MIP $\leq 60 \%$ of predicted), it was suggested the realization of inspiratory muscle training (IMT) with an inspiratory resistor device linear load. The initial setting was charge with $65 \%$ of MIP, for 6 weeks, daily, with 30 breaths performed twice daily. At 21 and 42 days were carried out new evaluations of MIP and VC, the BDI being reevaluated in 42 days. After 21 days the program an increase of $13.8 \%$ was seen in MIP and $82.3 \%$ in the CV. Already at 42 days, it was observed plus a gain in MIP without changing the values of CV. Conclusion: In the analysis of BDI was found an increase of 7 to 12 points (71.4\%), which features a no dyspnea patient to carry out the activities proposed by the instrument. It identified an improvement in muscle strength and improvement of dyspnea in daily activities after the TMI program in this case report.
\end{abstract}

Keywords: Sickle cell anemia; Lung function; Inspiratory muscle training

\section{INTRODUÇÃO}

\begin{abstract}
A Anemia Falciforme (AF) é uma doença hereditária autossômica recessiva, que é caracterizada por uma mutação do gene da cadeia beta da hemoglobina, resultando em alteração da hemoglobina normal ( $\mathrm{HbA}$ ) para uma hemoglobina falciforme com característica de foice (HbS). ${ }^{(1)}$ Essa polimerização da hemoglobina promove oclusão vascular, isquemia e infarto tecidual, com implicações sistêmicas, como as complicações pulmonares, que podem ser de natureza aguda ou crônica. ${ }^{(1-2)}$
\end{abstract}

As significantes alterações na função pulmonar a longo prazo podem ocorrer por consequência do comprometimento estrutural da caixa torácica, devido episódios de isquemia óssea nas costelas, progredindo para a diminuição da capacidade ventilatória e até restrição pulmonar³. Esse evento afe- ta a elasticidade do pulmão pela substituição do parênquima pulmonar por tecido fibrótico, podendo ser um fator determinante para a redução da reserva ventilatória e limitação para atividades da vida diária. ${ }^{(1,4)}$

Um dos aspectos que também podem estar associados a redução da reserva respiratória é a fraqueza muscular inspiratória, sendo que o treinamento muscular inspiratório pode ser uma forma de tratamento. Neste artigo, os autores descreveram os efeitos do treinamento muscular inspiratório na função pulmonar e status funcional em um indivíduo com anemia falciforme, que tinha queixas de dispnéia durante as atividades de vida diária e reinternamentos hospitalares frequentes. 


\section{RELATO DE CASO}

Paciente J.A.S., gênero masculino, 52 anos, aposentado por invalidez, com diagnóstico de anemia falciforme obtido na fase da adolescência e com histórico recorrente de atendimentos de emergência em unidade hospitalar devido as complicações da anemia falciforme. O paciente faz parte da Associação Baiana das Pessoas com Doença Falciforme (ABADFAL) e faz consultas frequentes no ambulatório, sem fazer parte de um programa de reabilitação. O mesmo relatou que tinha "sensação de falta de ar" ao realizar atividades da vida diária com ou sem esforços e durante as crises álgicas sentia muita dificuldade para respirar, sem relato de tabagismo.

A partir dessa queixa de dispnéia em algumas atividades de vida diária e a possível influência da fraqueza muscular inspiratória, foi proposto a realização do TMI a nível domiciliar, já que apesar da dispnéia, o mesmo conseguia realizar suas atividades de forma mais lenta. Para ser realizado o (TMI) se fez necessário uma avaliação prévia da força muscular inspiratória. Esta que ocorreu por meio da mensuração da pressão inspiratória máxima $\left(\mathrm{PI}_{\text {maxx }}\right)$, através do aparelho POWERbreathe ${ }^{\circledR}$ digital modelo $\mathrm{K}_{5}$ (POWERbreathe International Limited, Londres, Inglaterra). As mensurações foram realizadas com o paciente na posição sentada em uma cadeira, utilizando-se um clipe nasal para evitar escape aéreo, sendo que o mesmo foi orientado a realizar uma expiração máxima ao nível do volume residual, e em seguida uma inspiração máxima ao nível da capacidade pulmonar total. Esta manobra foi executada por três vezes, onde a melhor medida encontrada foi escolhida como parâmetro antes do treinamento. Na mesma incursão respiratória, além da $\mathrm{PI}_{\text {máx' }}$ foi verificado a capacidade vital $(C V)$, já que o equipamento avalia esses dois itens de forma simultânea.

Como a $\mathrm{PI}_{\text {máx }}$ e a CV não são capazes de predizer de forma isolada algum grau de capacidade funcional mais uma ferramenta de avaliação foi realizada, o índice basal de dispnéia (BDI). Este índice avalia o grau da dispnéia nas atividades da vida diária com um escore que varia de o a 12 pontos, tendo como indicadores três categorias: prejuízo funcional (atividades de vida diária cotidianas sem dispnéia), magnitude da tarefa (atividades extraordinárias como carregar cargas muito pesadas no plano, cargas mais leves ao subir ladeiras, escadas ou correndo sem dispnéia) e magnitude do esforço ("falta de ar" com o maior esforço imaginável, sem apresentar falta de ar com esforços ordinários). Quanto menor a pontuação total, pior a gravidade da dispnéia. ${ }^{(5)}$

$\mathrm{Na}$ avaliação inicial foram obtidos os valores de $\mathrm{PI}_{\text {máx }}=-36 \mathrm{cmH}_{2} \mathrm{O}$ e $\mathrm{CV}=1,7 \mathrm{~L}$ (tabela 1), além do BDI com um escore total de 7 pontos (tabela 2). A partir dessas aferições foi programado o TMI utilizando o aparelho POWERbreathe ${ }^{\circledR}$ modelo Classic Light Resistance (POWERbreathe International Limited, Londres, Inglaterra), com uma carga ajustada em $65 \%$ da $\mathrm{PI}_{\text {máx }}$. O paciente foi orientado a realizar trinta respirações com o aparelho de $\mathrm{TMI}$, duas vezes ao dia (uma pela manhã e outra pela noite), durante os sete dias da semana (Figura 1). O programa teve uma duração total de seis semanas e o paciente não relatou desconforto ou dificuldade em utilizar o dispositivo. 


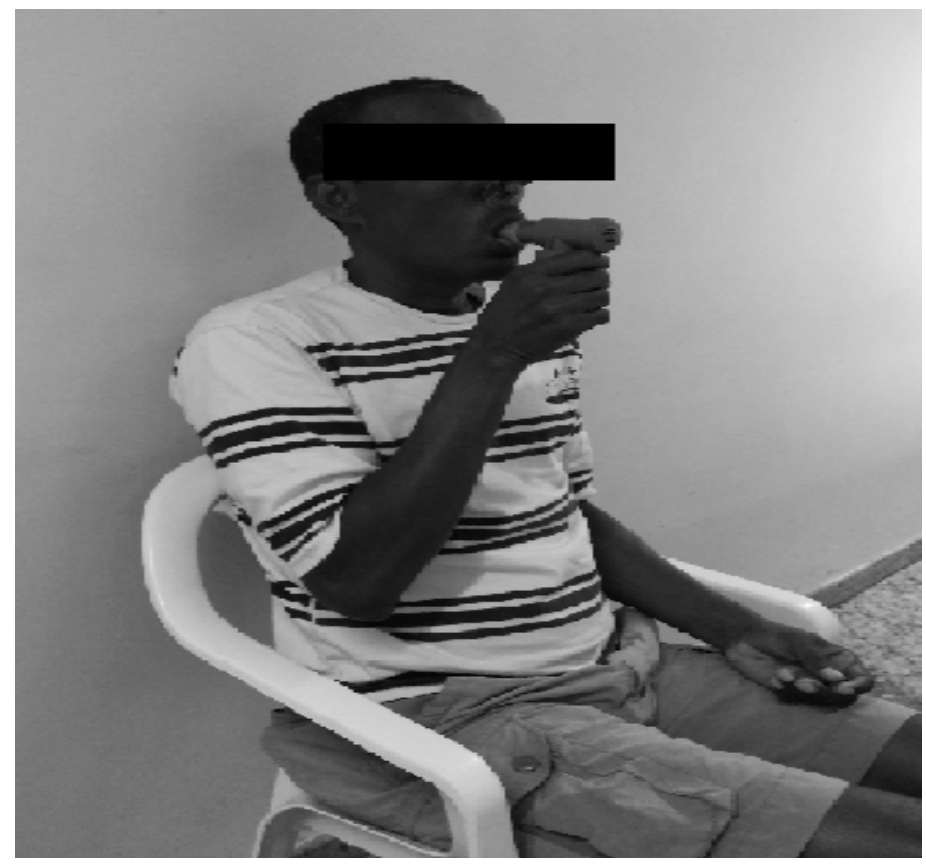

Figura 1 - Execução do treinamento muscular inspiratório.

Tabela 1 - Valores obtidos nas avaliações da $\mathrm{PI}_{\text {máx }}$ e da CV.

\begin{tabular}{cccc}
\hline & 1a aVALIAÇÃo & 2a AVALIAÇÃo & 3a AVALIAÇÃo \\
\hline $\mathrm{PI}_{\text {máx }}$ & $-36 \mathrm{cmH}_{2} \mathrm{O}$ & $-41 \mathrm{cmH}_{2} \mathrm{O}$ & $-74 \mathrm{cmH}_{2} \mathrm{O}$ \\
$\mathrm{CV}$ & $1,7 \mathrm{~L}$ & $3,1 \mathrm{~L}$ & $3,1 \mathrm{~L}$ \\
\hline
\end{tabular}

$\mathrm{PI}_{\text {máx }}=$ pressão inspiratória máxima; $\mathrm{CV}$ =capacidade vital; $\mathrm{cm} \mathrm{H}_{2} \mathrm{O}=$ centímetros de água; $\mathrm{L}=$ litros.

Tabela 2 - Resultados do índice basal de dispnéia por categoria.

\begin{tabular}{lcc}
\hline \multicolumn{1}{c}{ Categoria } & BDI adMissão & BDI aPós 42 Dias de tRatamento \\
\hline Prejuízo funcional & Grau 3 & Grau 4 \\
Magnitude da tarefa & Grau 2 & Grau 4 \\
Magnitude do esforço & Grau 2 & Grau 4 \\
\hline
\end{tabular}

$\mathrm{BDI}=$ I Índice basal de dispnéia.

No $21^{\circ}$ dia do tratamento foi realizada uma nova avaliação, onde foi verificado elevação de 13,8\% na $\mathrm{PI}_{\text {máx }}$ e 82,3\% na CV (tabela1). Neste mesmo dia, a carga do resistor linear foi reajustada com base no novo valor de $\mathrm{PI}_{\text {máx }}$ mantendo-se em $65 \%$ da força gerada pela musculatura inspiratória do indivíduo. Ao final do programa, no $42^{\circ}$ dia, foi realizada a última avaliação da função pulmonar, sendo observado um ganho na $\mathrm{PI}_{\text {máx }}$ de $80,4 \%$ em relação à $2^{\mathrm{a}}$ avaliação (21 dias de tratamento) e um ganho total de 105,5\% em relação ao estágio inicial. Já a capacidade vital, manteve o valor entre a $2^{\mathrm{a}}$ e última avaliação. Quando analisado o índice basal de dispnéia, houve um incremento de 7 para 12 pontos $(71,4 \%)$ no total, o que segundo a escala caracteriza um paciente sem dispnéia ao realizar as atividades propostas pelo instrumento (tabela 2). $\mathrm{O}$ paciente não relatou desconforto ao realizar o TMI e relatou não ter tido internações no período. 


\section{DISCUSSÃO}

Estudos comprovam que a realização do TMI é eficaz em melhorar a força muscular inspiratória em pacientes acometidos por diversas patologias, ${ }^{(6,7)}$ porém ainda não foi descrito na literatura o TMI em pacientes com $A F$, principalmente associado a melhora da dispnéia durante as atividades de vida diária como visualizado nesse relato de caso. A força muscular inspiratória reduzida semelhante a apresentada pelo paciente deste relato de caso, já tinha sido evidenciado em um estudo de Ohara e outros, ${ }^{(8)}$ em que mulheres com AF apresentaram valores de $\mathrm{PI}_{\text {máx }}$ abaixo do previsto no grupo avaliado, justificando assim a realização de TMI para ganho de força e endurance da musculatura inspiratória para este grupo de indivíduos ${ }^{8-9}$. Nesse relato de caso, o indivíduo apresentava um valor de força de aproximadamente $40 \%$ do valor predito para equação brasileira de referência, ${ }^{(10)}$ o que confirma a presença de fraqueza muscular inspiratória. ${ }^{(1)}$ Outros estudos ${ }^{(6,7,12)}$ também já apontaram que o uso de aparelho impondo uma determinada carga a musculatura inspiratória para treinamento favoreceu o ganho de força e endurance justificado pelo aumento da $\mathrm{PI}_{\text {máx }}$.

Durante a realização do TMI nas 6 semanas, observou-se um aumento da $\mathrm{PI}_{\text {máx }}$ para $45,5 \%$ do predito no $21^{\circ}$ dia e para $82,2 \%$ do predito no $42^{\circ}$ dia. Já a CV não seguiu o avanço proporcional da força muscular inspiratória, o que pode ser justificado pelo padrão restritivo de alteração estrutural na caixa torácica de pacientes falcêmicos, que apresenta caráter aparentemente de pouca reversibilidade. Essa relação não proporcional dessas duas variáveis, onde houve aumento contínuo da $\mathrm{PI}_{\text {máx }}$ nos dois momentos e na CV apenas no $1^{\circ}$ momento não é comum em todas situações. Em um estudo realizado com pacientes de pós-operatório de revascularização do miocárdio por exemplo, ${ }^{(13)}$ o grupo que realizou o TMI com o Threshold ${ }^{\circledR}$ Inspiratory Muscle Trainer (IMT) apresentou um ganho diretamente proporcional entre a $\mathrm{PI}_{\text {máx }}$ e a CV. Uma das justificativas para isso é que o transtorno na parede torácica nestes pacientes cirúrgicos é ape- nas devido ao ato cirúrgico e não estrutural como nos indivíduos com anemia falciforme.

O ganho da $\mathrm{CV}_{\text {e }} \mathrm{PI}_{\text {máx }}$ de forma isolada não prediz necessariamente uma melhoria na qualidade de vida do indivíduo, porém o resultado do TMI em resposta a dispnéia relatada durante as atividades, evidenciada no BDI, gera um resultado satisfatório que pode ter influência na vida deste indivíduo. Desde 2002, Romer e outros ${ }^{(14)}$ já haviam descrito a melhora da performance de ciclistas após TMI, entretanto, repercussões em indivíduos com algum quadro patológico ainda não são tão frequentes. Tuner e outros ${ }^{(15)}$ em 2013 foram um dos poucos que relataram a melhora da sensação de dispnéia em pacientes asmáticos submetidos a um protocolo de TMI.

Diferentemente, o TMI vem sendo utilizado com maior frequência em pacientes com insuficência cardíaca e Doença Pulmonar Obstrutiva Crônica (DPOC). Granville e outos ${ }^{(6)}$ identificaram que os pacientes com insuficiência cardíaca obtiveram uma redução da sensação de dispnéia e aumento na tolerância ao esforço associado ao ganho de força muscular inspiratória, após realizarem o TMI pelo período de 12 semanas. Já Gosselink e outros ${ }^{(16)} \mathrm{em} 2011$, descreveram em uma metanálise, que o TMI aumentou a força muscular respiratória e também a capacidade de exercício, semelhante ao presente relato de caso.

Um aspecto importante é que apesar das alterações sistêmicas da anemia falciforme, houve melhora da força muscular respiratória reduzida e minimização da sensação de dispnéia durante os esforços, semelhante a outras condições relatadas como asma e insuficiência cardíaca. Os achados desse estudo sugerem que esta técnica seja testada em populações maiores para avaliação de sua efetividade e possível implementação nos protocolos de reabilitação nessa população. Estudos randomizados e controlados são necessários para testar essa hipótese, já que esses resultados podem contribuir para uma melhora da qualidade de vida e ter influência na redução de reinternações hospitalares. 
O estudo apresenta limitações como o fato de ser um relato de caso, no qual não é possível excluir algumas variáveis de confundimento existentes. Outra limitação é que o paciente não realizou um programa de reabilitação associada, pelo fato de ser independente funcional para atividades, apesar da dispnéia.

Concluiu-se nesse relato de caso que o TMI teve influência na melhora da fraqueza muscular respiratória e aumento dos valores da CV no indivíduo com anemia falciforme, de forma associada à meIhora da dispnéia durante realização das atividades da vida diária. Estudos com tamanhos amostrais maiores são necessários para retirada de fatores de confundimento, bem como para avaliação de variáveis adicionais como a qualidade de vida.

\section{REFERÊNCIAS}

1. Moreira GA. Repercussões respiratórias da anemia falciforme. J Bras Pneumol. 2007;33(3):18-20.

2. Gualandro SFM, Fonseca GHH, Gualandro DM. Complicações cardiopulmonares das doenças falciformes. Rev Bras Hematol Hemoter. 2007;29(3):291-8.

3. Fonseca CSV, Melo CAA, Carvalho RM, Neto JB, Araújo JG, Cipolotti R. Função pulmonar em portadores de anemia falciforme. Rev Paul Pediatr. 2011;29(1):85-90.

4. Colman ML, Beraldo PC. Estudo das variações de pressão inspiratória máxima em tetraplégicos, tratados por meio de incentivador respiratório, em regime ambulatorial. Fisioter Mov. 2010 jul/set;23(3):439-49.

5. Mahler DA, Weinberg DH, Wells CK, Feinstein AR. The measurement of dyspnea. Contents, interobserver agreement, and physiologic correlates of two new clinical indexes. Chest. 1984;85:751-8.

6. Granville DD, Grunewald PG, Leguisamo CP, Calegari L. Treinamento muscular inspiratório em pacientes com insuficiência cardíaca: estudo de caso. Fisioter Pesq. 2007;14(3):62-8.
7. Souza LMC, Nogueira IDB, Guerra RO, Ferreira GMH, Nogueira PAMS. Influência do treinamento muscular inspiratório em idosas hipertensas com fraqueza muscular inspiratória. ConScientiae Saúde. 2014;13(1):86-92.

8. Ohara DG, Ruas G, Walsh IAP, Castro SS, Jamami M. Lung function and six-minute walk test performance in individuals with sickle cell disease. Braz J Phys Ther. 2014 JanFeb;18(1):79-87.

9. Zanoni $C T$, Rodrigues $C M C$, Mariano D, Suzan ABBM, Boaventura LC, Galvão F. Efeitos do treinamento muscular inspiratório em universitários tabagistas e não tabagistas. Fisioter Pesq. 2012;19(2):147-52.

10. Costa D, Gonçalves HA, Lima LP, Ike D, Cancelliero KM, Montebelo MIL. Novos valores de referência para pressões respiratórias máximas na população brasileira: Correções. J Bras Pneumol. 2010;36(5):667.

11. Caruso P, Albuquerque ALP, Santana PV, Cardenas LZ, Ferreira JG, Prina E, et al. Métodos diagnósticos para avaliação da força muscular inspiratória e expiratória. J Bras Pneumol. 2015;41(2):110-123.

12. McConnell AK, Lomax M. The influence of inspiratory muscle work history and specific inspiratory muscle training upon human limb muscle fatigue. J Physiol. 2006;577: 445-457.

13. Matheus GB, Dragosavac D, Trevisan P, Costa CE, Lopes MM, Ribeiro GCA. Treinamento muscular melhora o volume corrente e a capacidade vital no pós-operatório de revascularização do miocárdio. Rev Bras Cir Cardiovasc. 2O12;27(3):362-9.

14. Romer LM, McConnell AK, Jones DA. Effects of inspiratory muscle training on time-trial performance in trained cyclists. Journal of Sports Sciences. 2002;20:547-62.

15. Tuner LA, Mickleborough TD, McConnell AK, Stager JM et al. Effect of Inspiratory Muscle Training on Exercise Tolerance in Asthmatic Individuals. Med \& Scien in Sports \& Exer 2011;11:2031-38.

16. Gosselink R, De Vos J, Van Den H, Segers J, Decramer M, Kwakkel G. Impacto f inspiratory training in patients with COPD: what is the evidence? Eur Respir J. 2011; 37(2):233-5. 